\title{
GW23-e1086 INTERRELATION AMONG THE SERUM LEUKOTRIENE B4 LEVEL, ARACHIDONATE 5-LIPOXYGENASE ACTIVATING PROTEIN GENE SG13S89G/A POLYMORPHISM AND RISK OF ACUTE MYOCARDIAL INFARCTION
}

doi:10.1136/heartjnl-2012-302920k.25

He Guo-Ping, Ye Shan, Hui Jing-Jiao, Qi Chuan-Ping, He Guo-Ping. Affiliated Wujin

Hospital of Jiangsu University, Changzhou 213002, China

Objectives To investigate the interrelation among the serum leukotriene (LT) B4 level, the arachidonate 5-lipoxygenase activating protein (ALOX5AP) gene SG13S89G/A polymorphism and the risk of acute myocardial infarction (AMI) in the Chinese Han population of Changzhou region.

Methods The polymorphism in the ALOX5AP gene SG13S89G/A was genotyped by PCR and restriction fragment length polymorphism analysis and the serum LTB4 level (M/IOR) was measured by ELISA in 262 patients with AMI (AMI group) and 132 subjects with chest pain who were free from coronary heart disease by coronarography (control group).

Results As compared with those in control group, there were no significant differences in (AA+GA) and GG genetype $(7.58 \%$ vs $4.96 \%$ and $92.42 \%$ vs $95.04 \%$, respectively), and A allele frequencie (4.17\% vs $2.67 \%$ ) of ALOX5AP gene SG13S89G/A locus in AMI group (all the $\mathrm{p}$ value $>0.05$ ). Multivariable logistic regression analysis showed that there was no significant association between ALOX5AP gene SG13S89G/A polymorphism and the risk of AMI. The serum LTB4 level in AMI group was significantly higher than the one in control group (477.97/370.52 pg/ml vs 200.57/ $236.65 \mathrm{pg} / \mathrm{ml}, \mathrm{p}<0.001)$. Multivariable logistic regression analysis show that the serum LTB4 level was significantly correlated with the risk of AMI. No significant difference in serum LTB4 levels was found between (AA+GA) and GG genetype in AMI group or control group (all the $\mathrm{p}$ value $>0.05$ ).

Conclusions The serum LTB4 level is significantly increased in patients with AMI; the ALOX5AP gene SG13S114G/A polymorphism may not be correlated to the risk of AMI and not influence the serum LTB4 level in Chinese Han population of Changzhou region. 